\title{
Structure and Orientation Determination of Metal-Oxide Nanostructures by Electron Backscatter Diffraction
}

\author{
Y.N. Picard, ${ }^{*}$ L. Mazeina, ${ }^{*}$ S. Maximenko, ${ }^{*}$ J.A. Freitas, ${ }^{*}$ S.M. Prokes, ${ }^{*}$ and M.E. Twigg* \\ *Electronics Science and Technology Division, Naval Research Laboratory, Washington, DC 20375
}

Crystal growth directions, epitaxial relationships, and identities of crystallographic surfaces are all fundamental parameters that will influence novel electronic, chemical, mechanical or optical behavior in nanostructures. Electron backscatter diffraction (EBSD) stands as a robust approach for rapidly determining the crystal structure and orientation of nanostructures. However, aside from a few recent studies, including analysis of GaAs [1] and GaN [2] nanowires, EBSD has not been widely applied towards studying nanostructures.

In this study, EBSD is utilized to investigate the structure and orientation of metal-oxide nanostructures. Nanowires (NWs) and nanoribbons are grown on various substrates via the vapordeposition approach. Kikuchi patterns are recorded using a commercial EBSD system housed inside a scanning electron microscope operating at 10 or $20 \mathrm{keV}$.

Vertical Sn-O NWs grown on c-plane $\mathrm{Al}_{2} \mathrm{O}_{3}$ substrates with Au catalyst, shown in Fig. 1, exhibit the rutile $\mathrm{SnO}_{2}$ structure as determined by EBSD. The vertical nature of these $\mathrm{SnO}_{2} \mathrm{NWs}$ allows direct comparisons to the known substrate surface plane. The analysis consistently shows that these NWs grow along the [100] direction and exhibit an epitaxial relationship where the $\mathrm{SnO}_{2}(100)$ plane is parallel to the $\mathrm{Al}_{2} \mathrm{O}_{3}(0001)$ plane, and the $\mathrm{SnO}_{2}$ [010] and [001] directions are parallel to the $\mathrm{Al}_{2} \mathrm{O}_{3}$ [1210] and [1010] directions, respectively [3]. This EBSD approach is also employed to determine the growth direction and exposed surfaces of $\mathrm{SnO}_{2}$ nanoribbons.

EBSD can also be extremely complimentary to other techniques, such as cathodoluminescence (CL) spectroscopy. A CL-based real-color imaging technique developed to monitor the luminescence characteristics of nanostructures has been applied to image unique Ga-Sn oxide heterostructures, shown in Fig. 2. Optical properties are inhomogeneous along the heterostructure length ranging from intense blue emission, peak-centered at $430 \mathrm{~nm}$, to broad red emission at $600 \mathrm{~nm}$. According to electron dispersive spectroscopy (EDS), these heterostructures show a conversion in cation composition from Ga-dominant to Sn-rich, with O nearly evenly distributed throughout. Changes in optical and chemical properties directly correlate with the results of spot EBSD analysis. The Garich, blue emission phase is monocrystalline $\beta-\mathrm{Ga}_{2} \mathrm{O}_{3}$, while the red luminescent regions are identified as polycrystalline rutile $\mathrm{SnO}_{2}$.

The observation of orientation variation along these nanostructure lengths, as well as optimal SEM voltage and current parameters for EBSD analysis of different nanostructure diameters is discussed.

References

[1] S.V. Prikhodko et. al., Ultramicroscopy 109 (2008) 133.

[2] J.P. Long et. al., Nano Lett. 7 (2007) 831.

[3] L. Mazeina, Y.N. Picard et. al., submitted. 

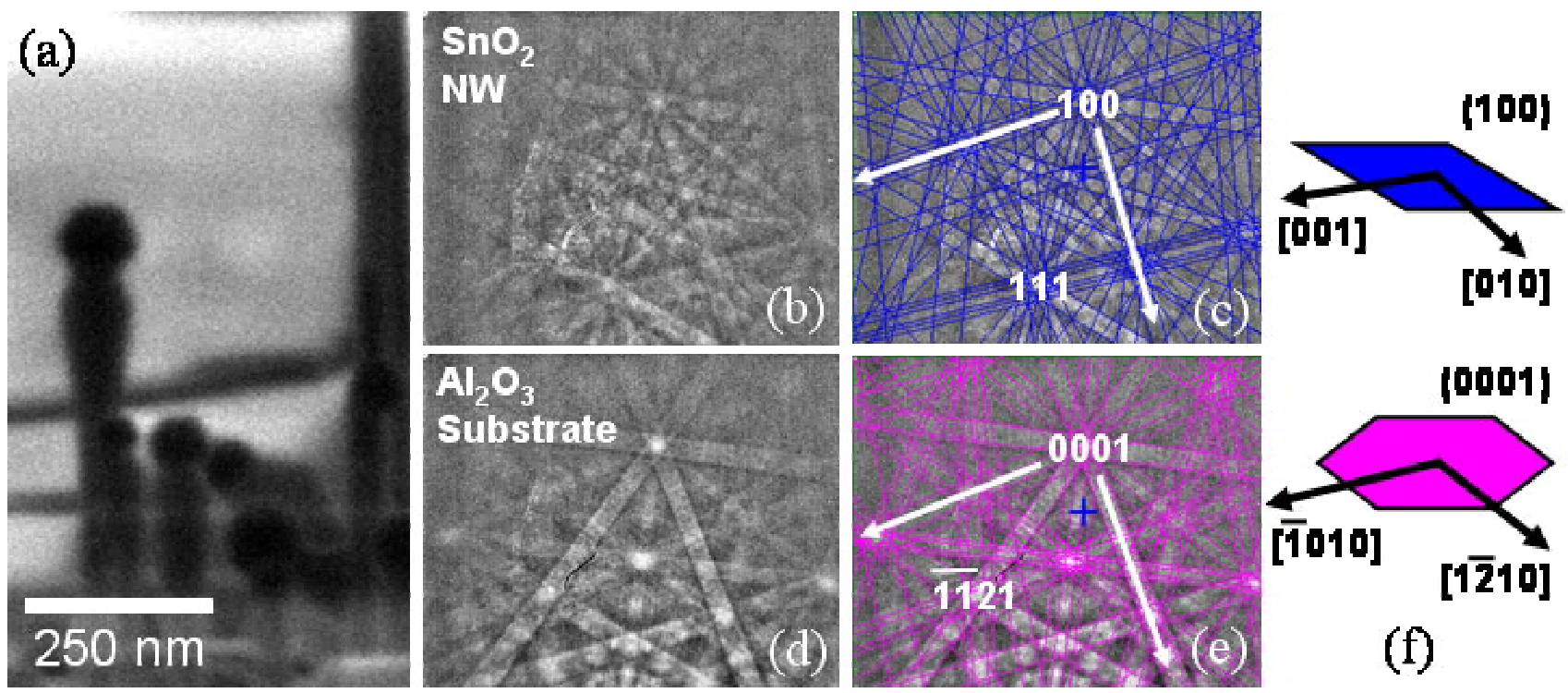

(f)

Fig. 1. Vertical $\mathrm{SnO}_{2}$ nanowires grown on c-plane $\mathrm{Al}_{2} \mathrm{O}_{3}$ substrates imaged by (a) SEM. EBSD analysis confirms the nanowires are (b-c) rutile $\mathrm{SnO}_{2}$ and also indicates an epitaxial relationship to the (d-e) corundum $\mathrm{Al}_{2} \mathrm{O}_{3}$ substrate. This epitaxial relationship (f) can be summarized as $\mathrm{SnO}_{2}(100)$ $\| \mathrm{Al}_{2} \mathrm{O}_{3}$ (0001) with $\mathrm{SnO}_{2}$ [001],[010] \| $\mathrm{Al}_{2} \mathrm{O}_{3}$ [1010],[1210].

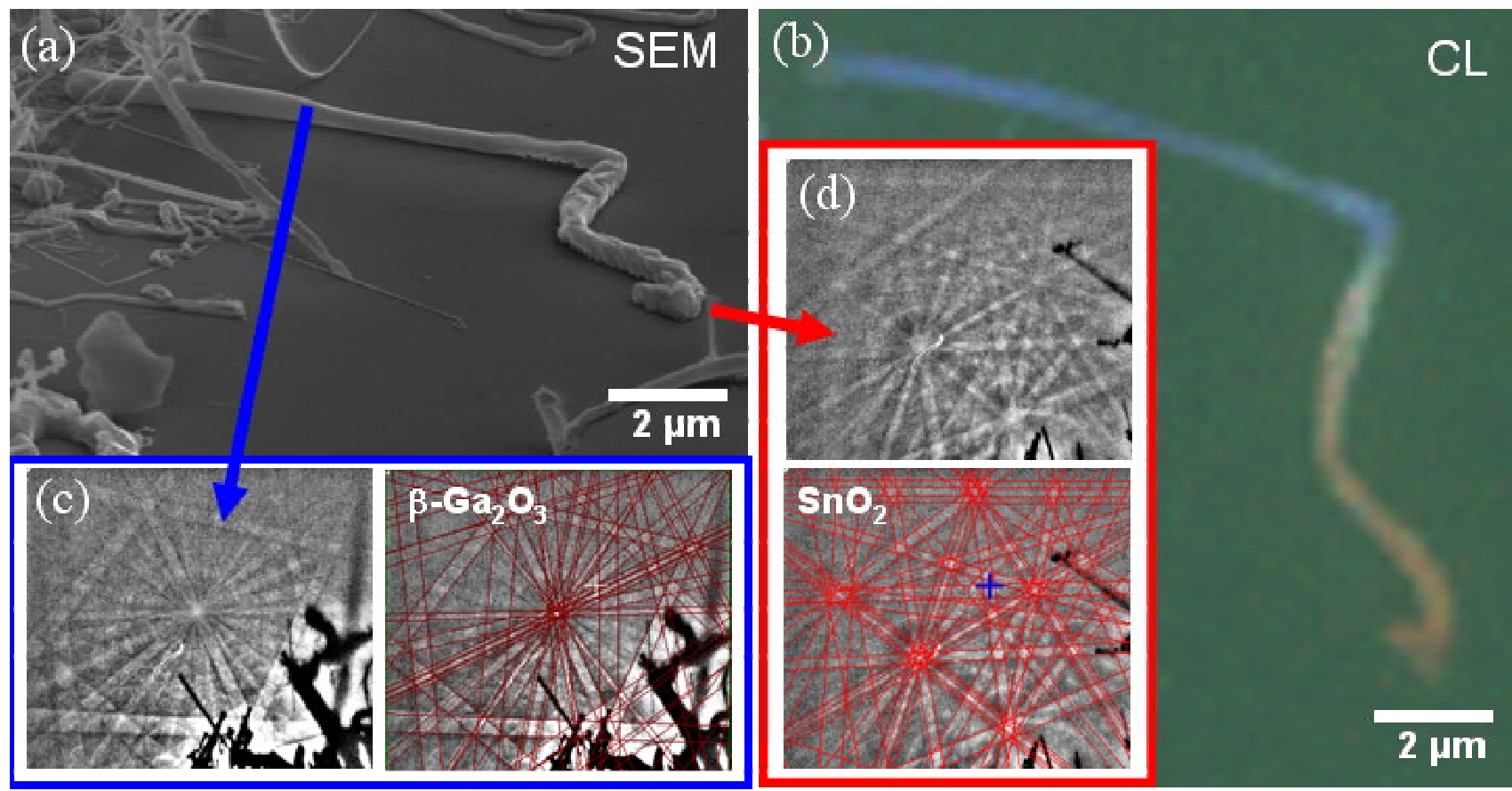

Fig. 2. A Ga-Sn oxide nanostructure imaged by (a) SEM and (b) cathodoluminescence. The blue luminescent portion of the nanoscale heterostructure is Ga rich according to EDS and exhibits (c) the monoclinic $\beta-\mathrm{Ga}_{2} \mathrm{O}_{3}$ crystal structure according to spot EBSD analysis. The opposite end of the heterostructure is red luminescent, Sn rich according to EDS analysis, and exhibits (d) the rutile $\mathrm{SnO}_{2}$ crystal structure according to spot EBSD analysis. 